\title{
Artículo Original: Lesión tisular debida a dispersión térmica por el uso de electrodos monopolares
}
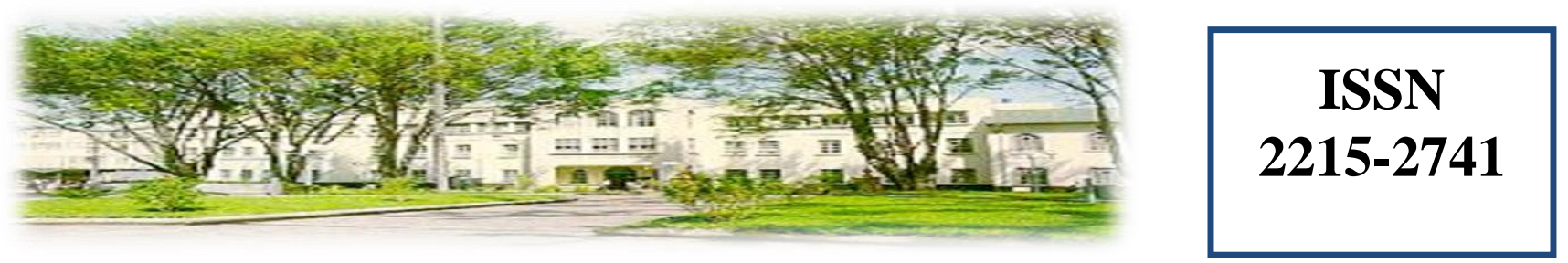

Hospital San Juan de Dios, San José, Costa Rica. Fundado en 1845

Recibido:

$16 / 05 / 2016$

Aceptado:

$15 / 06 / 2016$

Gonzalo Azúa Córdova ${ }^{1}$

Marco Zúñiga Montero ${ }^{2}$

Dennis Chaves Chaves ${ }^{3}$

José Luis Quirós Alpízar ${ }^{4}$

\begin{abstract}
${ }^{1}$ Especialista en Urología, Coordinador Académico de LEICIMI. Laboratorio de Entrenamiento e Investigación en Cirugía Mínimamente Invasiva. Universidad de Costa Rica, San José, Costa Rica. E-mail: ceicimi.em@ucr.ac.cr

${ }^{2}$ Especialista en Cirugía General. Director de LEICIMI. Laboratorio de Entrenamiento e Investigación en Cirugía Mínimamente Invasiva. Universidad de Costa Rica, San José, Costa Rica.

${ }^{3}$ Médico General, profesor asistente de LEICIMI. Laboratorio de Entrenamiento e Investigación en Cirugía Mínimamente Invasiva. Universidad de Costa Rica, San José, Costa Rica.

${ }^{4}$ Especialista en Patología, profesor Departamento de Anatomía Universidad de Costa Rica. Laboratorio de Entrenamiento e Investigación en Cirugía Mínimamente Invasiva. Universidad de Costa Rica, San José, Costa Rica.
\end{abstract}

\section{RESUMEN}

El uso de unidades de electrocirugía permite disección, corte y hemostasia debido al calor generado por el flujo de la corriente eléctrica a través del tejido, efecto denominado diatermia. Este calor, se difunde en un fenómeno conocido como dispersión térmica al tejido vecino, pudiendo lesionarlo. Comparadas con otras modalidades, las unidades monopolares son las que producen mayor lesión tisular por dispersión térmica y dentro de las diferentes modalidades de uso que permiten estas unidades es el modo de coagulación el que produce mayor daño tisular.

En el presente estudio se investiga la extensión de la lesión en el tejido que se produce por dispersión 
térmica con el uso de 3 diferentes electrodos monopolares ( 2 utilizados en cirugía abierta y 1 en cirugía laparoscópica) en la modalidad de coagulación a una potencia de $35 \mathrm{~W}$ en un modelo de hígado porcino ex vivo. La extensión del daño tisular se estableció midiendo la distancia de los cambios histológicos ocasionados luego de la utilización de cada electrodo. La extensión lateral y en profundidad de la lesión histológica fue de 0,82 y $0,82 \mathrm{~mm}, 0,81$ y $0,81 \mathrm{~mm}, 0,86$ y $0,88 \mathrm{~mm}$ con el electrobisturi \#1, \#2 y \#3 respectivamente. En conclusión, no hubo diferencia significativa en la extensión de la lesión histológica con el uso de los diferentes electrodos monopolares.

\section{PALABRAS CLAVE}

Dispersión térmica. Monopolar. Electrocirugía.

\section{ABSTRACT}

The use of electrosurgical units allows dissection, cutting and hemostasis due to heat generated by the flow of electrical current through the tissue, also known as the diathermy effect. This heat diffuses in a phenomenon known as thermal dispersion to surrounding tissue, and the tissue could be injured. If compared with other modalities, monopolar units are those that produce greater tissue damage due to thermal dispersion and among these units' different modes of use, coagulation mode is the one that produces more tissue damage. This study researches the extent of injured tissue produced by thermal dispersion when using three different monopolar electrodes ( 2 used in open surgery and one during laparoscopic surgery), in the coagulation mode at a power of $35 \mathrm{~W}$ in a porcine liver model, ex vivo. The extent of tissue damage was established by measuring the distance of the histological changes caused after the use of each electrode. The histological lateral extension and depth of the lesion was 0.82 and $0.82 \mathrm{~mm}, 0.81 \mathrm{~mm}$ and $0.81,0.86$ and $0.88 \mathrm{~mm}$ with electro-scalpel \# 1 , \# 2 and \# 3 respectively.
In conclusion, there was no significant difference in the extension of the histological lesion when using different monopolar electrodes.

\section{KEY WORDS}

Thermal dispersion. Monopolar. Electrosurgery

\section{INTRODUCCIÓN}

La utilización de equipos de electrocirugía ha significado un avance importante en el desarrollo de la cirugía a nivel mundial, que se ha traducido en beneficios para el paciente con un menor tiempo operatorio y menor sangrado transoperatorio. Existen diferentes tecnologías para la utilización de electrocirugía, sin embargo se calcula que más del $90 \%$ de las cirugías se realizan con equipos monopolares, lo cual aplica también a la cirugía laparoscópica. Esta modalidad de electrocirugía ha sido comparada con otras tecnologías más modernas y en general se ha encontrado que, si bien es altamente efectiva, comparativamente produce mayor daño al tejido circundante por la dispersión térmica lateral y eventualmente mayor riesgo de lesiones transoperatorias inadvertidas.

Se han realizado algunos estudios para valorar y comparar la extensión de la lesión por dispersión térmica lateral entre diferentes instrumentos quirúrgicos: diatermia monopolar y bipolar, bisturí harmónico y fusión de tejidos, encontrando que el mayor daño tisular lo producen los instrumentos monopolares 1, 4, 5, 7,15 siendo este mayor cuando se utiliza en la modalidad de coagulación ${ }^{2,16}$.

Por otro lado, para la realización de la cirugía laparoscópica, se han desarrollado instrumentos, incluidos electrobisturíes, que permitan realizar los procedimientos con la misma seguridad que en cirugía abierta. Sin embargo, los electrodos utilizados son más largos, de diferente forma y con una superficie de contacto con el tejido distinto, por lo que podrían presentar diferencia en cuanto a la extensión de la lesión tisular por dispersión 
térmica con respecto a instrumentos utilizados en cirugía abierta.

No se encuentran estudios que comparen el daño tisular lateral producido por los diferentes electrodos monopolares y por ende los hallazgos con electrodos de cirugía abierta no se pueden extrapolar a la cirugía laparoscópica. El objetivo de este estudio es comparar la extensión de la lesión tisular debido a la dispersión térmica lateral y en profundidad que se produce con el uso de diferentes instrumentos monopolares en el tejido biológico, tomando como modelo el hígado de cerdo. Así se pondrá establecer si es similar entre los electrodos utilizados en cirugía abierta y laparoscopía.

\section{MATERIALES Y MÉTODOS}

\section{Material biológico}

Se utilizó el hígado de un cerdo sano, sacrificado con fines comerciales en las 12 horas previas al experimento. Todos los cortes se realizaron en el mismo hígado.

\section{Unidad de electrocirugía}

Generador monopolar marca ValleyLab ${ }^{\circledR}$ modelo Force FX-CS. 110-120 voltios, de 390 kHz (Figura 1).

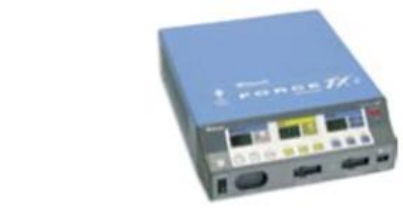

\section{Force FX-CS}

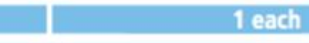

Figura 1: Unidad de electrocirugía monopolar Fuente: Valleylab, 2002

\section{Electrodo activo (bisturí)}

Se utilizaron 3 bisturíes. Electrobisturí \#1: Lápiz para electrocirugía monopolar con conexión de 3 pines, con electrodo de acero inoxidable, punta tipo espátula de $2.4 \mathrm{~mm}$ (código E2515 del catálogo Covidien). Electrobisturí \#2: Lápiz para electrocirugía monopolar con conexión de 3 pines, con electrodo de acero inoxidable, punta tipo espátula de $2.4 \mathrm{~mm}$, recubierto de silicón con propiedades elastoméricas (código $\mathrm{E} 2350 \mathrm{H}$ del catálogo de Covidien). Electrobisturí \#3: Electrodo sólido laparoscópico para energía monopolar de $36 \mathrm{~cm}$ de longitud con punta en forma de gancho y diámetro de 4.8 mm (código E2783-36 del catálogo de Covidien) (Figura 2).

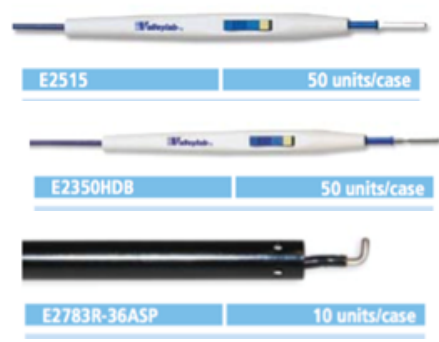

Figura 2. Electrodos monopolares. Fuente: Valleylab, 2002

\section{Electrodo de retorno (placa)}

Se utilizó un electrodo de retorno marca Valley$\mathrm{Lab}^{\circledR}$ con tecnología tipo REM, con hidrogel, tamaño pediátrico (código E7510-25 del catálogo de Covidien) recomendado para pacientes de 6 a 30 libras. Superficie conductiva de contacto no menor a $75 \mathrm{~cm}^{2}$ (Figura 3 ).

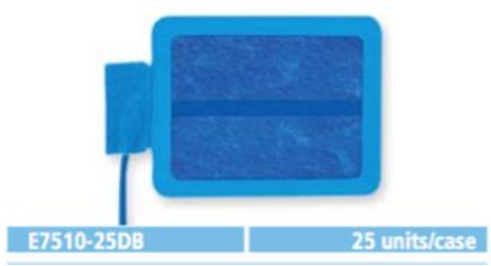

Figura 3. Electrodo de retorno. Fuente: Valleylab, 2002

\section{Procedimiento}


Se realizaron 30 incisiones en el hígado porcino de $2 \mathrm{~cm}$ de longitud y $1 \mathrm{~cm}$ de profundidad con cada uno de los diferentes electrodos (Fig. 4). Para la incisión se utilizó la modalidad de coagulación a una potencia de $35 \mathrm{~W}$ y todas las realizó el mismo cirujano.

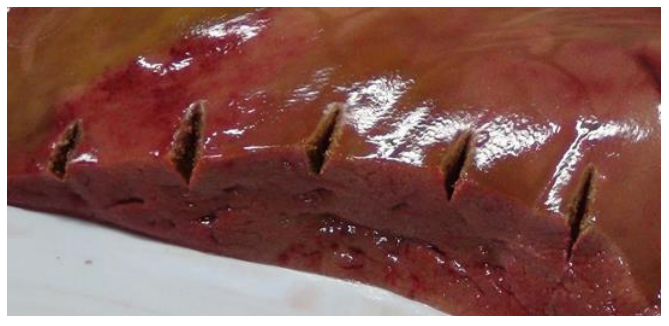

Figura 4. Incisiones con electrodo monopolar en hígado porcino.

Fuente: Valleylab, 2002

\section{Procesamiento de las muestras}

Los cortes histológicos fueron procesados para posterior tinción con hematoxilina y eosina de acuerdo con procedimiento del Instituto de Patología de las Fuerzas Armadas de los Estados Unidos de América (AFIP) ${ }^{13}$. Las láminas fueron enmascaradas para la revisión del daño tisular por el patólogo.

\section{Determinación del daño tisular}

La evaluación histológica de todas las muestras fue realizada por un mismo patólogo con un microscopio Olympus BX50 con escala milimétrica. Se tomó como daño tisular, la banda eosinofílica que se genera a partir del punto donde se aplica el electrocauterio, que es similar a la necrosis coagulativa que produce cariorexis y cariólisis de los núcleos de los hepatocitos. Se realizaron 3 mediciones para definir la extensión del daño tisular: márgenes laterales y margen profundo. Se tomó como el borde libre de corte en $\mathrm{V}$, como inicio de la medición, trazando una línea perpendicular hasta la zona más cercana en la cual se reconozcan células hepáticas conservadas, con núcleos claramente definidos. (Figuras 5 y 6 )

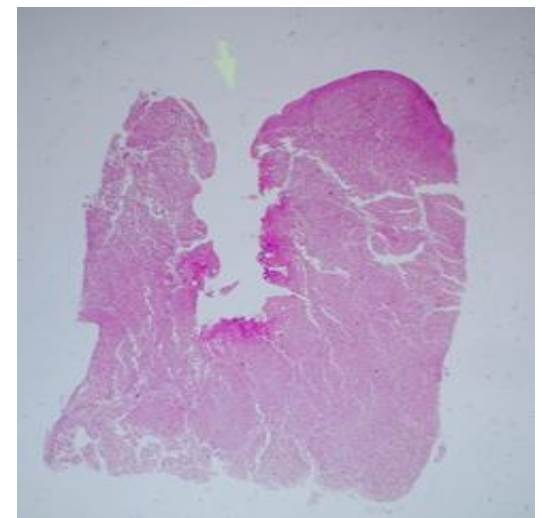

Figura 5. Corte histológico con defecto por incisión con electrodo monopolar (flecha).

Fuente: LEICIMI

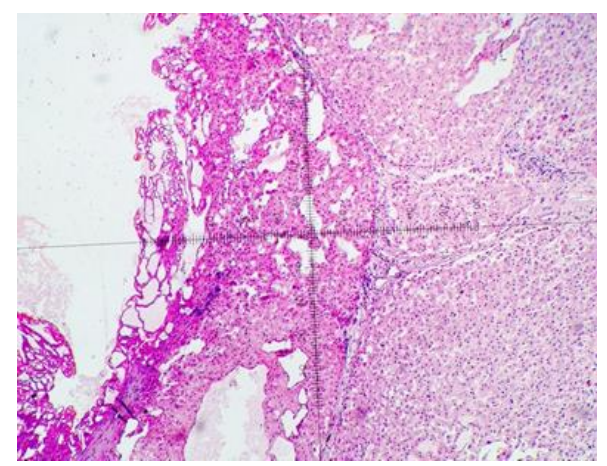

Figura 6: Corte histológico: medición del daño tisular

Fuente: LEICIMI

\section{Análisis estadístico}

Se utilizó análisis de varianza de bloques aleatorizados (ANOVA) cuya hipótesis nula plantea que la afectación con los tres métodos (electrodos) es igual y la hipótesis alternativa plantea que al menos 1 es diferente.

\section{RESULTADOS}

De los 30 cortes realizados con cada uno de los electrodos, solo pudieron ser evaluados 29, 26 y 
27 del bisturí 1, 2 y 3 respectivamente para el estudio del daño lateral y 24, 21 y 25 para el estudio del daño en profundidad, debido a problemas técnicos en el procesamiento de las muestras

La extensión del daño lateral del tejido en ambos sentidos (derecho e izquierdo) fue de 0,82 y 0,82 ; 0,82 y 0,$79 ; 0,83$ y $0,88 \mathrm{~mm}$ para el bisturí 1,2 y 3 respectivamente.

La distribución del daño tisular debido a la dispersión térmica lateral tanto del lado izquierdo como derecho del electrodo muestra una distribución normal, sin que existan diferencias estadísticamente significativas con $\mathrm{p}=0.969$ y $\mathrm{p}=0.092$ respectivamente.

En cuanto a la profundidad se observó un daño tisular de $0,82 \mathrm{~mm} ; 0,81 \mathrm{~mm}$ y $0,88 \mathrm{~mm}$ con el bisturí 1, 2 y 3 respectivamente. La extensión del daño tisular en profundidad debido a la dispersión térmica muestra una distribución normal, cuya media se encuentra en el intervalo $[0,8232$, 0,9365] con un nivel de confianza del $95 \%$. No existen diferencias estadísticamente significativas en los promedios de la dispersión térmica en profundidad entre los electrodos $(\mathrm{p}=0,248)$

\section{DISCUSIÓN}

Dada la amplia utilización de equipos electroquirúrgicos y su importancia en la cirugía es necesario conocer la extensión del daño que produce en el tejido, con el fin de que el cirujano pueda maximizar su utilidad y disminuir el riesgo por su uso.

Dado que el uso de diatermia monopolar genera la mayor temperatura en el tejido y por ende una marcada dispersión lateral, el diseño experimental utilizado en este estudio buscó determinar la extensión del daño tisular debido a la dispersión térmica comparando el efecto producido por electrodos monopolares utilizados en cirugía abierta y laparoscópica en el modo de coagulación.
De acuerdo con los resultados obtenidos, la extensión de dicho daño fue similar con los tres electrobisturíes utilizados, produciendo lesión del tejido vecino tanto lateral como en profundidad en un rango de extensión entre 0,81 y $0,88 \mathrm{~mm}$.

Para estudios posteriores, donde se valore el daño por dispersión térmica en otros tejidos, es importante tener en cuenta que con los diferentes electrodos monopolares se presentaron lesiones similares a una misma potencia.

\section{CONCLUSIONES}

La extensión del daño tisular por dispersión térmica es similar con los tres electrodos monopolares utilizados en el estudio.

\section{BIBLIOGRAFÍA}

1. Alkatout I, Schollmeyer T, Hawaldar N.A, Sharma N y Mettler L: Principles and safety measures of electrosurgery in laparoscopy. Journal of the Society of Laparoendoscopic Surgeons. JSLS 16:130-139, 2012.

2. Carus T, R. K. (2011). Collateral tissue damage by several types of coagulation (monopolar, bipolar, cold plasma and ultrasonic) in minimally invasive, perfused model. International Scholarly Research Network ISNR Surgery .

3. Dodde R, Gee JS, Geiger J.D y Shih: Monopolar electrosurgical thermal management for minimizing tissue damage. IEEE Transactions on biomedical engineering. 59 (1): 167173, 2012.

4. Druzijanic N, P. Z. (2012). Comparison of lateral thermal damage of the human peritoneum using monopolar diathermy, Harmonic scalpel and Ligasure. . Canadian Jurnal Surgery, 55 (5), 317-321.

5. Hefermehl L, L. R. (2014). Lateral temperature spresd of monopolar, bipolar and ultrasonic instruments for robot-assisted laparoscopic surgery. . BJU Int , 114 (245252), 2014. 
6. Humes D, Ahmed I y Lobo D: The Pedicle Effect and Direct Coupling: Delayed Thermal Injuries to the Bile Duct After Laparoscopic Cholecystectomy. Arch Surg 145: 9698,2010 .

7. Ko R, T. A. (2009). Comparison of the thermal and histopathological effects of bipolar and monopolar electrosurgical resection of the prostate in a canine model. BJU Int , 105, 1314-1317.

8. Law KS, Lyons DD: Comparative studies of energy sources in gynecologic laparoscopy. J Minim Invasive Gynecol, 20 (3): 308- 318, 2013.

9. Lyons S.D, Law K.S: Laparoscopic vessel sealing technologies. Journal Minimally Invasive Gynecology. JMIG 20: 301-307, 2013.

10. Massaarweh N, Cosgriff $\mathrm{N}$ y Slakey DP: Electrosurgery: History, principles and current and futures uses. J Am Coll Surg, 202 (3): 520-530, 2006.

11. Odell R.C: Surgical complications specific to monopolar electrosurgical energy: Engineering changes that have made electrosurgery safer. Journal Minimally Invasive Gynecology. JMIG 20: 288-298, 2013.

12. Phillips CK, Hruby GW, Durak E, Lehman DS, Humphrey PA, Mansukhani MM, Landman J: Tissue response to surgical energy devices. Urology, 71 (4): 744-748, 2008.

13. Prophet E, M.B. (1994). Laboratory Methods in Histotechnology. Armed Forces Institute of Pathology (U.S. Washington, D,C: Editorial American Registry of Pathology.*

14. Soderstrom R.M: Histologic changes associated with electrosurgical injury. Journal Minimally Invasive Gynecology. JMIG 20 (3): 288-298, 2013.

15. Sutton P.A, A. S. (2010). Comparison of lateral thermal spread using monopolar and bipolar diathermy, the harmonic scalpel and the ligasure. . British Journal of Surgery , 97, 428-433.
16. Tulikangas P, S. T. (2001). Gross and histologic characteristics of laparoscopic injuries with four different energy sources. Fertility and Sterility, 75 (4), 806-810.

17. Vilos G.A, Rajakumar C: Electrosurgical generators and monopolar y bipolar electrosurgery. Journal Minimally Invasive Gynecology. JMIG 20 (3): 279-287, 2013.

CONFLICTO DE INTERÉS Y/O AGRADECIMIENTOS

Los autores declaran que no existió ningún conflicto de interés en el presente reporte.

\section{FUENTES DE APOYO}

No hay fuentes de apoyo que declarar. 


\section{ANEXOS}

Cuadro 1. Estadísticos descriptivos de la distancia $(\mathrm{mm})$ del daño tisular del borde derecho con cada electrodo

\begin{tabular}{|l|l|l|l|l|l|l|l|l|}
\hline & & & & & \multicolumn{2}{l|}{ IC para la media al 95\% } & & \\
\hline Electrodo & N & Media & $\begin{array}{l}\text { Desviación tí- } \\
\text { pica }\end{array}$ & $\begin{array}{l}\text { Error tí- } \\
\text { pico }\end{array}$ & $\begin{array}{l}\text { Límite in- } \\
\text { ferior }\end{array}$ & $\begin{array}{l}\text { Límite su- } \\
\text { perior }\end{array}$ & Mínimo & Máximo \\
\hline Bisturí 1 & 29 & 0,821 & 0,155 & 0,029 & 0,762 & 0,880 & 0,57 & 1,29 \\
\hline Bisturí 2 & 26 & 0,823 & 0,143 & 0,028 & 0,765 & 0,881 & 0,60 & 1,16 \\
\hline Bisturí 3 & 27 & 0,833 & 0,260 & 0,050 & 0,730 & 0,936 & 0,42 & 1,52 \\
\hline Total & 82 & 0,826 & 0,191 & 0,021 & 0,784 & 0,867 & 0,42 & 1,52 \\
\hline
\end{tabular}

Fuente: LEICIMI

Cuadro 2. Estadísticos descriptivos de la distancia $(\mathrm{mm})$ del daño tisular del borde izquierdo con cada electrodo

\begin{tabular}{|c|c|c|c|c|c|c|c|c|}
\hline \multirow[t]{2}{*}{ Electrodo } & \multirow[t]{2}{*}{$\mathbf{N}$} & \multirow[t]{2}{*}{ Media } & \multirow{2}{*}{$\begin{array}{l}\text { Desviación tí- } \\
\text { pica }\end{array}$} & \multirow{2}{*}{$\begin{array}{l}\text { Error } \\
\text { típico }\end{array}$} & \multicolumn{2}{|c|}{$\begin{array}{c}\text { IC para la media al } \\
95 \%\end{array}$} & \multirow[t]{2}{*}{ Mínimo } & \multirow[t]{2}{*}{ Máximo } \\
\hline & & & & & $\begin{array}{l}\text { Límite } \\
\text { inferior }\end{array}$ & $\begin{array}{l}\text { Límite su- } \\
\text { perior }\end{array}$ & & \\
\hline Bisturí 1 & 29 & 0,8207 & 0,155 & 0,029 & 0,7616 & 0,8798 & 0,57 & 1,29 \\
\hline Bisturí 2 & 26 & 0,8231 & 0,143 & 0,028 & 0,7653 & 0,8808 & 0,60 & 1,16 \\
\hline Bisturí 3 & 27 & 0,8333 & 0,260 & 0,050 & 0,7303 & 0,9357 & 0,42 & 1,52 \\
\hline Total & 82 & 0,8255 & 0,191 & 0,021 & 0,7836 & 0,8674 & 0,42 & 1,52 \\
\hline
\end{tabular}

Fuente: LEICIMI

Cuadro 3. Distancia $(\mathrm{mm})$ del daño tisular en pro-

fundidad con cada electrodo

\begin{tabular}{|c|c|c|c|c|c|c|c|c|}
\hline \multirow[b]{2}{*}{ Electrodo } & \multirow[b]{2}{*}{$\mathrm{N}$} & \multirow[b]{2}{*}{ Media } & \multirow[b]{2}{*}{$\begin{array}{l}\text { Desviación } \\
\text { típica }\end{array}$} & \multirow[b]{2}{*}{$\begin{array}{l}\text { Error tí- } \\
\text { pico }\end{array}$} & \multicolumn{2}{|c|}{ IC para la media al $95 \%$} & \multirow[b]{2}{*}{ Mínimo } & \multirow[b]{2}{*}{ Máximo } \\
\hline & & & & & $\begin{array}{l}\text { Límite in- } \\
\text { ferior }\end{array}$ & $\begin{array}{l}\text { Límite su- } \\
\text { perior }\end{array}$ & & \\
\hline Bisturí 1 & 24 & 0,8162 & 0,16772 & 0,03423 & 0,7454 & 0,8871 & 0,5 & 1,11 \\
\hline Bisturí 2 & 21 & 0,93 & 0,3091 & 0,06745 & 0,7893 & 1,0707 & 0,51 & 1,9 \\
\hline Bisturí 3 & 25 & 0,8988 & 0,22135 & 0,04427 & 0,8074 & 0,9902 & 0,41 & 1,35 \\
\hline Total & 70 & 0,8799 & 0,23751 & 0,02839 & 0,8232 & 0,9365 & 0,41 & 1,9 \\
\hline
\end{tabular}

Fuente: LEICIMI 
